K-electrons. Inserting these values in $(7) *$ shows that $\tan (\Delta \alpha / 2)$ is reduced by a factor of about 2.9

* The small difference in the value of the exponent $n$ has been neglected for simplicity.

1 G. Borrmann, Naturwiss. 42, 67 [1955].

2 G. Borrmann and H. Wagner, Naturwiss. 42, 68 [1955].

3 G. Borrmann, G. Hildebrandt, and H. Wagner, Z. Physik 142, 406 [1955].

4 K. Lehmann and G. Borrmann, Z. Kristallogr. 125, 1 [1967].

5 G. Borrmann, W. Hartwig, and H. Irmler, Z. Naturforsch. $13 \mathrm{a}, 423$ [1958]. when passing the absorption edge from the lowenergy side to the high-energy side.

${ }^{6}$ U. Bonse and M. Hart, Z. Physik 188, 154 [1965].

7 L. Gerward, Physica Scripta 4, 71 [1971].

8 A. R. Lang, Acta Cryst. 12, 249 [1959].

9 N. Kato, Acta Cryst. 13, 349 [1960].

10 G. Grimvall and E. Persson, Acta Cryst. A 25, 417 [1969].

11 E. Persson and O. N. Efimov, Phys. Stat. Sol. (a) 2, 757 [1970].

12 H. Wagenfeld, Phys. Rev. 144, 216 [1966].

\title{
The Borrmann Effect in Few-beam Electron Diffraction Patterns:
}

Use of the Born Series to Analyse Diffraction Asymmetries

\author{
P. Goodman
}

Division of Chemical Physics, CSIRO, Clayton, Victoria, Australia

(Z. Naturforsch. 28 a, 580-587 [1973] ; received 1 February 1973)

Dedicated to Professor G. Borrmann on the occasion of his 65th birthday

\begin{abstract}
A description is given of the Borrmann effect associated with a 3-and 4-beam interaction, as observed in the zero beam distribution of a Kossel-Möllenstedt electron diffraction pattern. In the 3-beam interaction which is studied in detail, the contribution to the intensity asymmetry is characterised by the order of the Born process. Considering the inversion symmetry about the central intersection, even order terms contribute an absorption effect, independent of the phase of the structure amplitude, while odd order terms contribute a structure dependen term which to the first order is independent of absorption. Considering the asymmetry across the weak line and parallel to the strong line, the absorption contributes an asymmetry which inverts with the sign of the structure amplitude. This latter property of the Borrmann effect has possible applications in structure analysis.
\end{abstract}

\section{Introduction}

The presence of a Borrmann ${ }^{1}$ Effect in electron diffraction was recognized at least as early as Honjo and Mihama's (1954) report on fine structure patterns from $\mathrm{MgO}^{2}$. Also many Kossel-Möllenstedt patterns showing asymmetries and other effects due to dynamic interactions with absorption were published, for example by Hoerni ${ }^{3}$. Since that time the complete n-beam dynamic theory has become available, so that the possible scope of an investigation is now very wide.

Quantitative examination of the Borrmann effect in electron diffraction can be made only by a detailed calculation of dynamic intensities, and for this purpose a fairly thorough knowledge of the structure is required. However, the recent renewed

Reprint requests to The Chief, Division of Chemical Physics, CSIRO, P.O. Box 160, Clayton, Victoria, Australien 3168. interest in electron diffraction of structurally. unknown compounds indicates that there may be also room for more analytical approaches to dynamic scattering. Qualitative analysis of the Borrmann effect in Kossel-Möllenstedt patterns, i. e., of zerobeam asymmetries, can be rewarding in tracing the origins of asymmetry, in studies of structure and absorption. Few-beam patterns discussed here can be obtained by choice of orientation from crystals of moderate unit-cell size and moderate thickness, but must be interpreted through the complete theory to obtain reliable conclusions.

\section{Theory}

In 1957 an analytical solution to the n-beam scattering problem for forward scattering from a crystal of finite thickness became available ${ }^{4}$. This was later given as a Born series of products ${ }^{5}$,

$$
U(h)=\sum_{n} E_{n}(h) \cdot Z_{n}(h)
$$


between a structure factor term, $E_{n}(h)$, and a geometric term $Z_{n}(h)$, itself a series. Alternative methods of analysis result from developments of Sturkey's matrix method ${ }^{6}$. Each method has advantages in different directions. Previously, special few-beam cases have been examined using the eigenvalue method, starting from fairly rigid pre-conditions ${ }^{7,8}$. This method has some advantages for particular experiments, but in general the simple form of the Born solution, and its freedom from a priori assumptions make it very useful, particularly in investigations of symmetry.

For the $n$th Born term the first part [Eq. (1)] is a product of the structure factors to the power of involvment in the scattering path,

$$
E_{n}(h)=E_{1}{ }^{k} \cdot E_{2}{ }^{l} \cdot E_{3}{ }^{m} \ldots
$$

with $\Sigma(k+l+m \ldots)=n$, and the second part $Z_{n}(h)$ is a homogeneous, oscillating series in powers of $\zeta_{h}$ (excitation errors) and $H$ (the crystal thickness). $E_{n}(h)$ therefore contains the influence of structure, and $Z_{n}(h)$ th influence of angle of incidence, or position in the Kossel-Möllenstedt diagram.

The 3-beam and 4-beam non-systematic interactions are the simplest that contain information on structure factor phases, in a readily accessible form in the Kossel-Möllenstedt pattern; the 2-beam case with absorption is included here for the sake of completeness and overall understanding.

\section{Two-Beam Diffraction with Absorption}

The general behaviour of the $Z_{n}$ functions can be found by studying a pair of functions for consecutive values of $n$.

As examples, the $Z$ functions for the 4 th and 5 th Born terms are:

$$
\begin{aligned}
& Z_{4}=+\frac{t^{4}}{4 !}+\frac{i t^{5}}{5 !}\left(2 \zeta_{h}\right)-\frac{t^{6}}{6 !}\left(3 \zeta_{h}^{2}\right)-\ldots, \\
& Z_{5}=+\frac{i t^{5}}{5 !}-\frac{t^{6}}{6 !}\left(3 \zeta_{h}\right)-\frac{i t^{7}}{7 !}\left({ }_{6}^{4} \zeta 9\right)+\ldots,
\end{aligned}
$$

where $t$ replaces $2 \pi H$ in the original reference ${ }^{5}$. Thus in even-order terms $(n=4)$, the change of sign of $\zeta_{h}$ affects the imaginary part, whereas in oddorder terms $(n=5)$ the real part is affected. This rule holds for all $n$, i. e., $Z_{n}$ has the form:

$$
\begin{aligned}
& Z_{n}=a{ }^{ \pm} i b \text { for } n \text { even } \\
& Z_{n}={ }^{ \pm} c+i d \text { for } n \text { odd } .
\end{aligned}
$$

For the (0)-beam to zero-order scattering term $Z_{0}(h) \cdot E_{0}(h)$, as pointed out in detail in reference 4, should be included. $Z_{0}(h)=1$, also $E_{0}(h)=1$. This invariant term, representing the directly transmitted incident wave, causes the special properties of the (0)-beam. [Here the bracketed indices refer to crystallographic indices, i.e., directions in reciprocal space, and these must be distinguished from the subscript of $Z$ and $E$ indices referring to the order of a particular scattering process. Thus (0)beam means, for a parallel-sided crystal, the whole beam transmitted in a direction parallel to the incident direction.] Thus,

$$
\begin{aligned}
\psi(0) & =1+\sum_{n} E_{n}(h) \cdot Z_{n}(h) \\
& =1+\sum_{n}(e+i f)\left(a^{ \pm} i b\right) \\
& =1+\sum_{n}(E+i F)\left(A^{ \pm} i B\right),
\end{aligned}
$$

where the capital letters contain a summation over all $n$. The alternative signs in front of $i B$ refer to positive and negative values of $\zeta_{h}$ respectively. It can be shown by multiplying out, and collecting real and imaginary parts for all $n$, that the step between the last two lines can be made. In this case only the $n$ even terms are included since in the 2-beam case only these contribute to the zero-beam scattering amplitude $\psi(0)$. The difference in intensities, $I=\psi_{(0)} \psi_{(0)}{ }^{*}$, between taking the + or sign in Equation (4), $I^{+}-I^{-}=4 B F$. This finite result comes from the cross products with the unity term. Similarly,

$$
\psi(h)=\left({ }^{ \pm} C+i D\right)(E+i F),
$$

which gives $I^{+}-I^{-}=0$. Asymmetry with respect to $\zeta_{h}$ arises therefore only in the $(0)$-beam, and then only with finite absorption, the asymmetry direction depending on the phase of the absorbing potential relative to the scattering potential. This result is otherwise known from the 2-beam expression in the well-known summed form.

\section{Three-Beam Diffraction without Absorption}

Contributions to the (0)-beam in the 3-beam case contain both $n$ even and $n$ odd terms, examples of which are shown in Figure 1. The same rules apply as before, viz.:

$$
\begin{array}{ll}
\sum_{n} Z_{n}=A^{ \pm} i B & \text { for } n \text { even, } \\
\sum_{n} Z_{n}={ }^{ \pm} C+i D & \text { for } n \text { odd }
\end{array}
$$




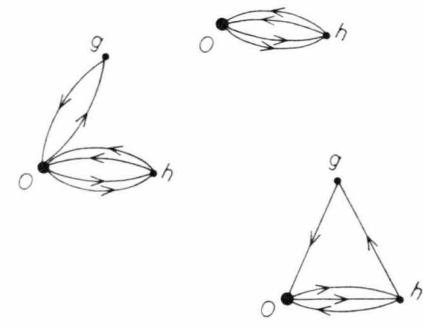

Fig. 1. Scattering Process leading to the (0)-beam. (middle) 2-beam. The 4th Born term,

(left) 3-beam. A 6th Born term ( $n$ even),

(right) 3-beam. A 5th Born term ( $n$ odd).

for the geometric term $Z_{n}(h)$. Here the change from + to - sign refers to a change of sign of the complete function, i. e., an inversion across the intersection centre of the 0-beam diagram, e.g., $(++)$ to $(--)$ or $(+-)$ to $(-+)$ quadrants in Figure 2. For example, for the case $n=5$ of Figure 1 (right)

$$
\begin{aligned}
Z_{5}=\frac{i t^{5}}{5 !}-\frac{t^{6}}{6 !}\left(2 \zeta_{h}+\zeta_{g}\right) & \\
& \quad-\frac{i t^{7}}{7 !}\left(3 \zeta_{h}{ }^{2}+\zeta_{g}{ }^{2}+2 \zeta_{h} \zeta_{g}\right) .
\end{aligned}
$$

At this stage the argument is general for any number of beams giving a common intersection in the Kossel-Möllenstedt diagram. For a rectangular section of reciprocal space all intersections are at least 4-beam. Since the 4- and 3 -beam problems give essentially similar results, the 3 -beam only is followed in detail. Actual choice for a particular problem depends upon the observation (see later discussion).

Amongst contributions to the $(0)$-beam in the 3 -beam case, only $n$-odd terms can be affected by change of phase of structure. Hence,

$$
E_{n}(h)=V_{h}{ }^{k} V_{g}^{l} V_{g-h^{m}} ; \quad n=k+l+m .
$$

For a centro-symmetric structure the $V$ 's are either all positive, or one is negative. Also $k, l, m$ are either all odd or all even, governed by the limited topologies for the 3 -beam case. Therefore, $E_{n}(h)$ inverts in sign for $n$ odd terms in going from one type of structure (all $V$ 's positive) to the other.
Taking the product $E_{n}(h) Z_{n}(h)$, for a non-absorbing structure, where $E_{n}(h)={ }^{ \pm} E$, by factoring out the $E$ we have the result shown in Table 1, for the two structures. Thus in going from one structure to the other the intensity will completely invert about the central intersection of the Kossel-Möllenstedt pattern, as indicated by the arrows in the Table 1. This conclusion comes without any approximation for the $n$-beam intersecting interaction without absorption.

\section{Three-Beam Diffraction with Absorption}

The simple result which gives an inversion of the diagram through its centre for the anti-phase structure is not one which is observed in practice. This is because we have omitted all other weak beam interactions, and absorption. In conditions where it is possible to isolate the few-beam interaction in the Kossel-Möllenstedt diagram, it can be shown that the weak beam interactions far from the diagram, i. e., of large excitation error, are represented to first order as an imaginary potential, or absorption. Very weak reflections which actually cross the diagram (pass through zero excitation error) also behave like an absorption near their line, with a small phase factor either side of the line; this latter is based on the assumption that very weak lines can be treated in a separate 3-beam interaction with the strong reflection, i. e., that products $V_{(g)}{ }^{\prime} \cdot V_{(g)}{ }^{\prime \prime}$ involving more than one weak term are negligible.

The influence of weak beams of large excitation error has been demonstrated using Sturkey's matrix formulation

$$
\psi_{(h)}=\exp \{i \sigma M\} .
$$

Partitioning the matrix and using Zassenhaus's theorum, the authors Anstis et al. ${ }^{9}$ show that

$$
\psi_{(h)} \approx \exp \left\{i \sigma M_{1}\right\} \exp \left\{-\sigma M_{2}\right\},
$$

where $M_{1}$ represents the few-beam matrix, and $M_{2}$ a matrix of weak beam terms; the second term has

Table 1 .

\begin{tabular}{lll}
\hline & $\zeta_{g}, \zeta_{h}$ positive & $\zeta_{h}, \zeta_{g}$ negative \\
\hline $\begin{array}{l}\text { All Structure Factors } \\
\begin{array}{l}\text { Positive } \\
\text { All Structure Factors } \\
\text { Negative }\end{array}\end{array}$ & $(1+A+C)+i(B+D)$ \\
\hline
\end{tabular}


the form of an absorption. Therefore, the simplest realistic treatment possible is the 3-beam interaction with absorption.

Substituting $E_{n}(h)=e+i f$, we have for the $(0)$. beam, following the procedure of the previous sections,

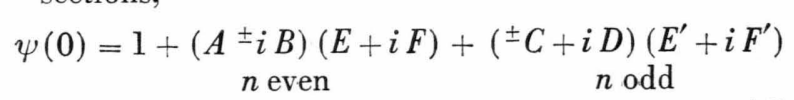

where $(E+i F)$ and $\left(E^{\prime}+i F^{\prime}\right)$ represent the complex structure factor products for the $n$ even and $n$ odd terms respectively. If we multiply out the intensities, and take $I^{+}-I^{-}$, the difference in intensities between quadrants related by inversion through the intersection point of the Kossel-Möllenstedt diagram, we obtain,

$$
\begin{aligned}
I_{(0)}{ }^{+}-I_{(0)}{ }^{-}=- & 4 B F \quad \text { (only } n \text { even terms) } \\
& +4 C E^{\prime} \quad \text { (only } n \text { odd terms) } \\
& +4\left(E \mathrm{E}^{\prime}+F F^{\prime}\right)(A C+B D)
\end{aligned}
$$

(cross product of $n$ even and $n$ odd terms).

The last two terms (viz.: $n$ odd only and the cross-product terms) will invert as the structure changes sign (a property of $E^{\prime}+i F^{\prime}$ ), but the first term, $-4 B F$, will not. $F$ contains all terms of the type $\left(V_{h}^{i}\right) \cdot\left(V_{g}\right)^{k} \cdot\left(V_{g-h}\right)^{l}$, of even order $n$, which have an absorption Fourier coefficient to the first order. Since it is generally true that $V_{h}{ }^{i} \ll V_{h}$ for any single reflection, we can say that the term $B F$ contains all the first order absorption effects. These terms come only from the $n$ even terms and are noninverting. Conversely, the inverting terms, to this approximation contain no absorption terms, when we neglect the product $F F^{\prime}$ as of second order.

Hence we obtain a separation in character between the two types of scattering process. This separation and the static term $4 B F$ explain one feature in Kossel-Möllenstedt patterns, namely that independent of relative phases of reflexions, the weak crossing lines remain darkest on the $\zeta_{h}$ negative side of the strong line. The observation that the phase effect causes a reversal of intensity across the weak line, rather than across the intersection as derived in the 3-beam case without absorption, was made without explanation in an earlier report ${ }^{10}$; this point is examined in more detail in Section 7 . The 3-beam type absorption effect must contribute to the asymmetry observed generally in KosselMöllenstedt and Kikuchi patterns with only very weak non-systematic reflections excited.

\section{Direction of Asymmetries and Comparison with Two-Beam Result}

To obtain a usuable result we must introduce two conditions, viz.: first that we have a crystal of moderate thickness, and secondly that $V_{h} \gg V_{g}$, $V_{g-h}$. The first condition means that the crystal should be of the order of a few hundred Anström units, i.e. at least a half of an extinction length thick with respect to the main reflection, but not extremely thick, as then the sensitive region of pattern shrinks too close to the reflection lines. The second condition allows us to terminate the series in the direction of high powers of $V_{g}, V_{g-h}$ and to place different topologies of high Born orders in order of importance. This condition is of practical value since it is possible to cross a main reflexion with several weak reflections in sequence, in order to determine their relative signs.

We cannot say $a b$ initio what the absolute signs of the collected terms $A, B, C, D$ from Eq. (4) to (7) of the previous sections are, since they represent oscillating convergent series. This agrees with the usual very complicated patterns obtained near many-beam interactions. However, under the conditions outlined above, the excitation error associated with the strong reflection is of special importance in determining the form of the pattern. This point will be examined later. We can determine the direction of the effect of the perturbation, keeping to the present method of investigation, by comparing individual terms of our series with the terms of the 2-beam series. The two relevant properties of the 2-beam solution are: (i) The influence of excitation error on the $(0)$-beam distribution. For a moderately thick crystal or for a substantial excitation error, as the excitation error decreases, the interaction increases and the intensity decreases (i. e. the intensity envelope of fringes in the KosselMöllenstedt pattern). (ii) With absorption the intensity is reduced relatively on the $\zeta_{h}$ negative side of the line. The region of the Kossel-Möllenstedt pattern, in terms of crystal thickness and excitation error, for which these statements are true is the region which we use in our observations of the Borrmann effect.

At first we must examine the direction of the effect in the 3-beam case without absorption. We must combine terms from the $n$ even and the $n$ odd series, which have different properties. It is sufficient to 
take two consecutive terms, e. g., $n$ and $n+1$ terms, since the total result may be obtained by repeated combinations of this type.

Taking the $n$ even and $n$ odd terms together in pairs, we find for example,

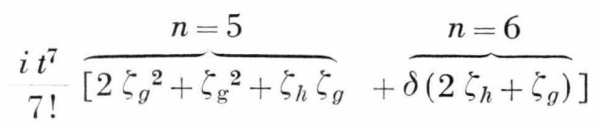

where $\delta=\frac{E_{n}(h)}{E_{n+1}(h)}= \pm \frac{\left|E_{n}(h)\right|}{\left|E_{n+1}(h)\right|}$, the (-) sign for the anti-phase structure, for the two most significant terms of the 5 th and 6 th Borm contributions with the common multiplier $-i \mathrm{t}^{7} / 7$ ! .

The reason for combining the two terms, of course, is to see how the two excitation error functions, one static and the other inverting with the double change of excitation error signs, interact. For, if we combine the complete series, term by term in this manner, we will obtain a new series with excitation error functions all behaving in a similar way with sign. In this way we obtain a series similar in form to the 2-beam series (i.e., one series for every second value of $n$ ) in which, apart from the different multiplying functions $E_{n}(h)$, we replace the 2 -beam excitation errors with a function involving $\zeta_{h}$ and $\zeta_{g}$. For all structure factors positive, $\delta$ is positive, so that if both $\zeta$ 's are of like sign the $\zeta_{h} \zeta_{g}$-positive quadrant will give a larger excitation error function than that for $\zeta_{h} \zeta_{g}$ negative. Thus for $\zeta_{h} \zeta_{g}$-negative we have the greater dynamic interaction and hence the lower (0)-beam intensity. The situation is reversed for the antiphase structure (one structure factor negative). For the change from the $(+-)$ quadrant to the $(-+)$ quadrant the same effect takes place but with reduced magnitude, since the effective value of the excitation error function is reduced.

The second problem is to obtain the direction of the absorption effect. We can compare with the known 2-beam result obtained by letting $\zeta_{g}$ approach $\infty$, far from the $g$ intersection. Then the $\zeta_{h}$ negative region has less intensity than the $\zeta_{h}$ positive region by absorption. In the 3 -beam case we need only consider the $n$ even terms for the absorption effect, thus making a comparison with the 2-beam case more direct. A characteristic of the 3-beam interaction with $V_{h} \gg V_{g}, V_{g-h}$ is that high Born terms of low power in $V_{g}, V_{g-h}$ are not negligible so that we have for example in the 12 th Born term an excita- tion error function $\left(6 \zeta_{h}+\zeta_{g}\right)$ in a significant term, compared with $\left(6 \zeta_{h}\right)$ in the 2 -beam case, i.e., we have present in the 3 -beam all paths of the 2-beam plus several others through the point $g$. Thus, firstly, changes of sign of $\zeta_{h}$ are more significant than changes of sign of $\zeta_{g}$. Secondly, if $\zeta_{g}$ is put $=0$ all excitation error functions are the same as in the 2-beam but there are many additional terms and therefore the interaction with the absorption is in the same direction as in the 2-beam case and is much greater. This is the main observable effect with regard to absorption, i. e., the Kossel-Möllenstedt pattern is generally darker on the $-\zeta_{h}$ side of the $h$ line, and particularly so along the line $\zeta_{g}=0$.

\section{Asymmetry Across the Weak Line}

Up till now we have only considered the symmetry by inversion through the pattern intersection, i.e., a simultaneous change of sign of both excitation errors; the intensity along the $\zeta_{g}=0$ falls into this category. In order to compare intensities on either side of a line, e. g., across the $g$ line in the $(0)$-beam distribution, [i.e. $(--)$ to $(-+)$ in Figure 2],

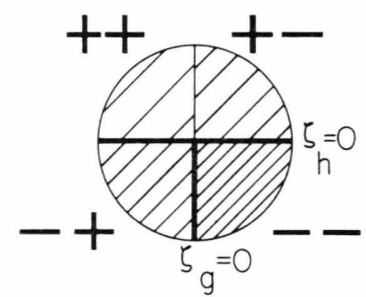

Fig. 2. Four quadrants of the (0)-beam Kossel-Möllenstedt pattern, formed by intersection of the lines at $\zeta_{h}=0, \zeta_{g}=0$. The quadrants are labelled by the signs of the two excitation errors; viz.: $(++)$ means $+\zeta_{h},+\zeta_{g}$ and is outside the two Kossel bands.

which is the most practical comparison experimentally, we must see the effect of changing the sign of either $\zeta_{h}$ or $\zeta_{g}$ alone. Using the previous termino$\operatorname{logy}$, viz.: $A+i B$ for $n$ even and $C+i D$ for $n$ odd processes, the influence of this sign change affects terms having even powers of $\zeta$ (i. e., $A$ and $D$ ) differently from those having odd powers of $\zeta$ (i. e., $B$ and $C$ ). The former terms are symmetrical with respect to change of $\zeta_{h}$ or $\zeta_{g}$, i. e., with respect to crossing $g$ or $h$ lines. The latter terms, for our special conditions, are much more influenced by change of $\zeta_{h}$.

If we work out the effect of crossing the weak $g$ lines, and list only those terms which remain signi- 
ficant, even for a weak reflexion $g$, we find for the (0) -beam intensity difference across the line:

$I^{+g}-I^{-g}=E A^{*}$

$$
-F^{\prime} D^{*}+A^{*} D^{*}\left(E^{\prime} F-E F^{\prime}\right),
$$

where ${ }^{*}$ indicates the influence on the excitation error terms of the change $+\zeta_{g} \rightarrow-\zeta_{g}$. The two latter terms are inverting with structure and contain absorption in the first order. Therefore, we can say that all first order absorption effects causing an asymmetry across the weak $\mathrm{g}$ line are structure inverting, and so contribute to the phase determination effect we are seeking to exploit. Absorption effects along the line $\zeta_{g}=0$ are non-inverting. This exactly fits all our previous observations. Two terms, viz. $E A^{*}$ and $\left(E A^{*}\right)^{2}$, are structurally static in this sense and also do not contain absorption to the first order.

The weight of terms which are structurally inverting and which are affected as much by crossing a weak line as a strong one explains the very marked (structure dependent) asymmetry which is observed in practice and caused by apparently very weak reflexions [e.g., see Fig. 3(c), reference ${ }^{11}$ ], and gives the power to the Borrmann effect method.

The remainder of the expression for $I^{+g}-I^{-g}$ consists of terms which have a small asymmetry in crossing a weak line but a large asymmetry in crossing a strong line. These are $B^{*} F$, the main absorption asymmetry term in crossing the $h$ line and which is static with respect to structure, plus the terms

$$
\begin{aligned}
& B^{*} C^{*}\left(E \mathrm{~F}^{\prime}-E^{\prime} F\right)+ \\
& \qquad E^{\prime}\left(C^{*} E^{*}+B^{*} D^{*}\right)+\left(E^{\prime} C^{*}\right)^{2},
\end{aligned}
$$

the first two of which invert in direction with phase of structure.

The conclusion is that the most sensitive place to observe the structural phase effect is on the $\zeta_{h}$ negative side of the $h$ line, at points relatively close to and either side of the $g$ line, rather than by attempting to compare points diametrically across the $g, h$ intersection, because of the overriding influence of the absorption asymmetry with respect to the $h$ line which has little sensitivity to structure.

Finally, we can consider the point $\zeta_{h}=0, \zeta_{g}=0$. For these values the whole Born series becomes identical to the "thin phase grating" 12 series which has no excitation error terms. The difference between the thin phase grating and the 3 -beam case here is due to the restricted topologies of the latter case. This illustrates the point that it is necessary to have the scattering diagrams in addition to the series in order to construct the few-beam cases correctly.

\section{Observation}

Two observations are provided. Both are from a rectangular section of reciprocal space and so are actually 4-beam interactions with $h, g,(g+h)$ reflections excited, and $(g-h)$ as the coupling reflection. The relevance of the weakest $(g+h)$ re-

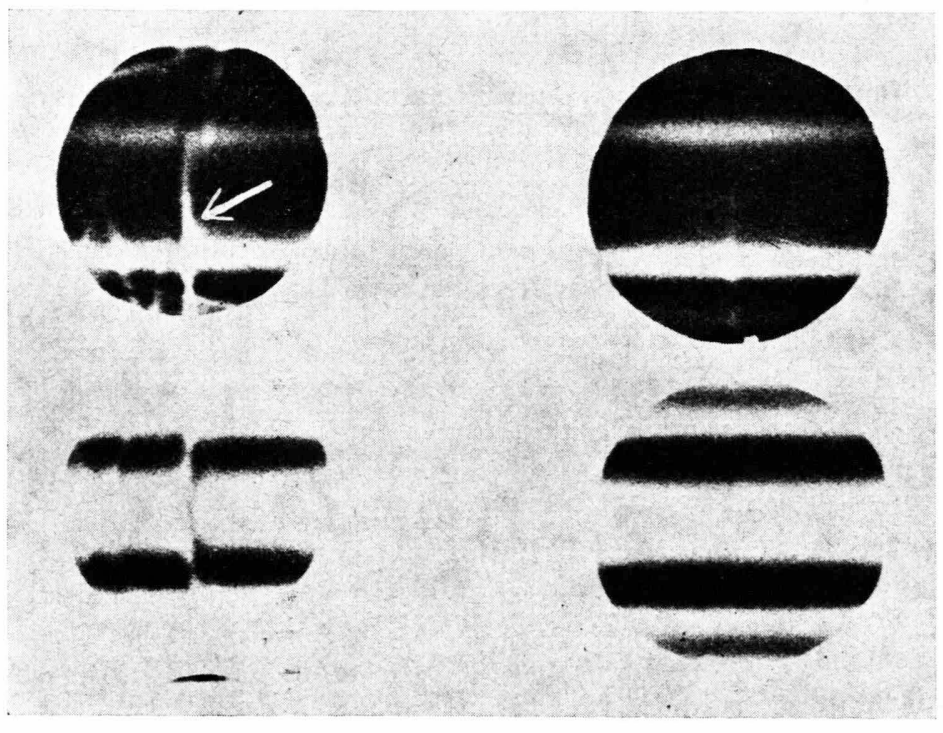

Fig. 3. Kossel-Möllenstedt patterns taken from $\mathrm{MgO}$ about the $002-820$ intersection at two temperatures, $300^{\circ} \mathrm{C}$ and $750^{\circ} \mathrm{C}$. An arrow on the $(0)$-beam, low temperature pattern indicates the absorption asymmetry about the weak (820) line, on the $\zeta_{002}$ negative side of the 200 line. This asymmetry, which is much clearer on the original photograph than on the reproduction, illustrates the phase effect with ratio for $V_{g} / V_{h}$ of only $1 / 10$. 


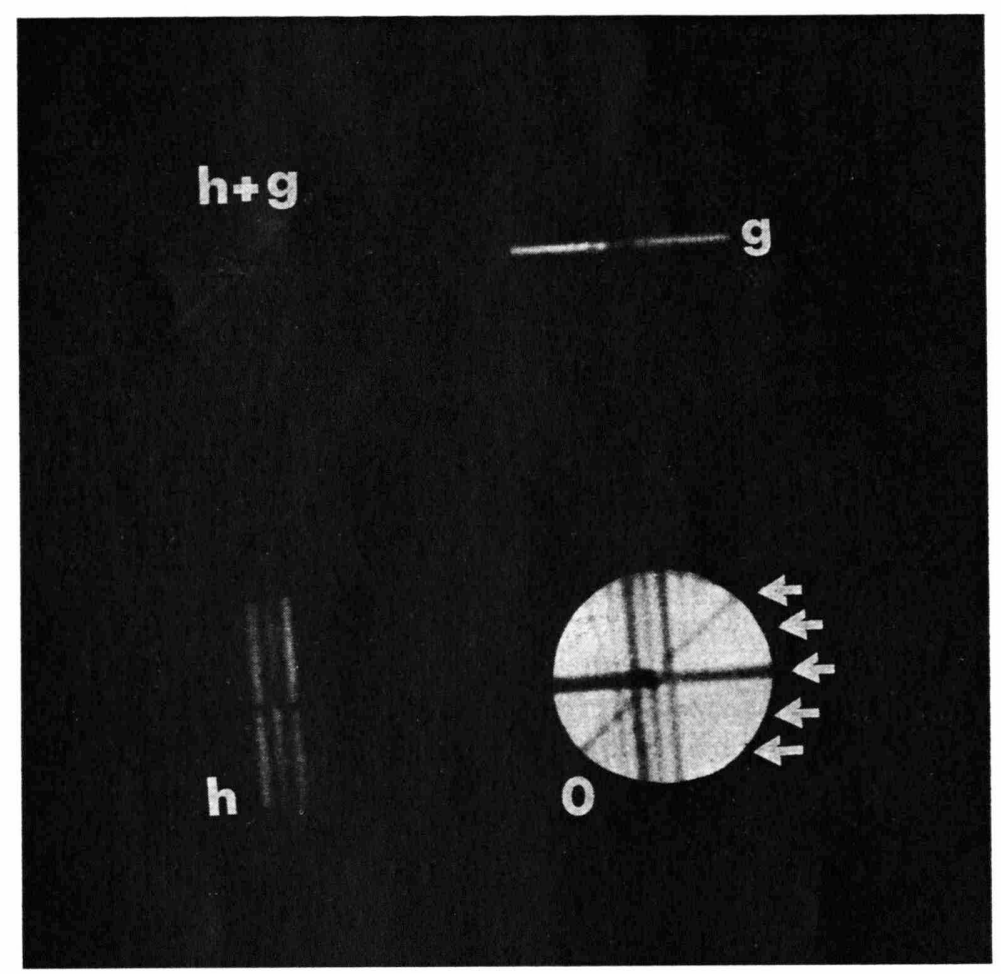

Fig. 4. Kossel-Möllenstedt pattern from graphite of the intersection of the $1 \overline{2} 12$, $20 \overline{2} 2$ and $3 \overline{2} \mathrm{~T} 4$ ( $h, g, h+g$, of the text) reflections. Photograph from the unpublished work of Dr. A. W. S. Johnson. With larger ratio for $V_{g} / V_{h}$ the region of interaction is wider than in Fig. 3, and covered by microphotometry of Fig. 5 along lines indicated by arrows.

flection can be tested by measuring the asymmetry it causes by division of the pattern; in both the patterns presented here the asymmetry is barely detectable in the (0)-beam. Hence for our present purpose we may as well treat these patterns with the 3-beam treatment outlined above.

(a) Two patterns from $\mathrm{MgO}$ are shown in Fig. 3 with the 002 and 820 reflection intersecting. Perturbation caused by the 820 is visible in Fig. 3 (left) at low temperature, with $V_{\mathrm{g}} / V_{h} \approx 0.12$, but not in 3 (right) with $V_{g} / V_{h}<0.1$. The darkest quadrant is $(--)$ i.e. $\zeta_{h}, \zeta_{g}$ negative. All structure factors are positive.

(b) Figure 4 shows the intersection of the $20 \overline{2} 2$ (g) reflections of graphite where the coupling reflection $(g-h)$ is $12 \overline{3} 0$, and the weak third reflection $(g+h)$ is $3 \overline{21} 4$. Microphotometer traces were taken across the $(0)$-beam from $\zeta_{h}$ positive to $\zeta_{h}$ negative. The five curves in sequence in Fig. 5 are for $\zeta_{g}$ negative (first two), $\zeta_{g}=0, \zeta_{g}$ positive (last two). The curves show the asymmetry to be greatest for $\zeta_{g}=0$, and greater for $\zeta_{g}$ positive than negative. The latter observation shows that absorption is greater for the $(-+)$ than for the $(--)$ quadrant. The two observations are to be expected for one reversed sign of structure factor as shown in
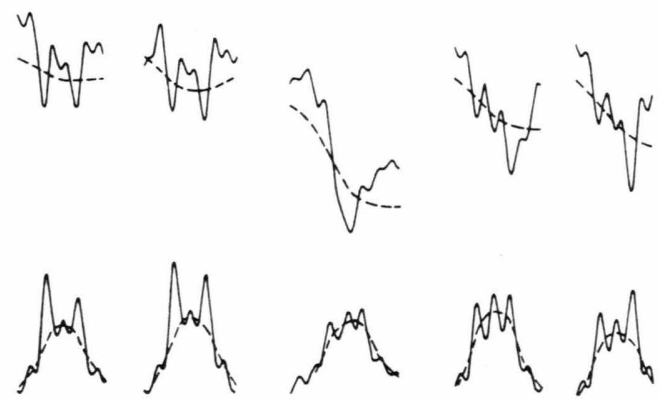

Fig. 5. Microphotometer traces taken from Fig. 4 of the (0)beam (upper half of diagram) along lines indicated by the arrows. Broken curves indicate the asymmetry. Lower half of diagram shows the collinear traces across the $(h)$-beam.

Table 2, combined with the absorption effect described above. Here the ratio $V_{g} / V_{h}$ is 0.2 .

Table 2. (in volts)

$\begin{array}{lllll}V_{h}-2.8 & V_{g}-0.55 & V_{g-h}+0.37 & V_{g+h}+0.28\end{array}$

\section{Application}

Here we have considered only centro-symmetric projections; so far as structure analysis is concerned non-centrosymmetric projections can be detected readily by the convergent beam method. However, 
these are complicated and best treated individually. By comparison with Kambe's ${ }^{7}$ method of phase determination from the detailed perturbation of the lines near the intersection, the method outlined here using the Borrmann effect does not require a large coupling structure factor $V_{g-h}$, and so is more generally applicable. The line perturbation described by Kambe can just be detected in Fig. 3 from graphite, but not at all in Figure 4 .

The more serious limitation with regard to applications is with respect to unit cell size. It does not seem possible to obtain Kossel-Mössenstedt (or Kikuchi) patterns from unit cells much in excess of $15 \AA$, giving distinct line intersections. Where lines have been obtained from large structures they are believed to have come from a sub-cell structure. For thin crystals with very large unit cells it would seem better therefore to use the thin phase grating approximation as the basis for qualitative methods.

1 G. Borrmann, Z. Phys. 42, 157 [1941].

2 G. Honjo and K. Mihama, J. Phys. Soc. Japan 9, 445 [1954].

3 J. Hoerni, Thesis, University of Geneva, 1950.

4 J. M. Cowley and A. F. Moodie, Acta Cryst. 10, 609 [1957].

5 J. M. Cowley and A. F. Moodie, J. Phys. Soc. Japan 17, 86, Supp. B 11 [1962].

${ }^{6}$ L. Sturkey, Proc. Phys. Soc. London 80, 321 [1962].
Another possible application lies in the observation of Kikuchi line intersections, particularly in specific loss patterns obtained by energy filtering. In this case it may be possible to determine the degree of localization in a structure of specific electrons, belonging to the structure.

\section{Acknowledgements}

The author wishes particularly to thank Dr. A. W.S. Johnson for suggestions for clarifying the original manuscript and for permission to publish Fig. 4 from his collection of hitherto unpublished work on graphite, and also Mr. A. F. Moodie for personal explanations of his theory and for pointing out alternative valuable approaches involving partitioning the scattering matrix. A further grateful acknowledgement is made to Dr. J. Gjønnes for valuable discussions on the subject of this paper.

7 K. Kambe, J. Phys. Soc. Japan 12, 13 [1957].

8 A. Fukuhara, J. Phys. Soc. Japan 21, 2645 [1966].

9 J. Anstis, D. F. Lynch, A. F. Moodie, and M. A. O'Keefe, Acta Cryst. A 29, 138 [1973]

10 P. Goodman, Acta Cryst. A 28, 92 [1971].

11 P. Goodman and G. Lehmpfuhl, Acta Cryst. 22, 15 [1967].

12 J. M. Cowley and A. F. Moodie, Acta Cryst. 12, 360 [1959]. 